\title{
Pediatricians must consider familial environment when diagnosing and managing childhood obesity
}

Young Suk Shim, MD

Department of Pediatrics, Ajou University Hospital, Ajou University School of Medicine, Suwon, Korea

\section{Key message}

- The prevalence of childhood obesity is increasing worldwide, including in the Republic of Korea, creating a major public health issue.

- Accumulated evidence indicates a strong relationship between parental and child obesity.

- A family-based approach is indicated to prevent and manage childhood and adult obesity.

Obesity is defined as the accumulation of excessive fat resulting from an imbalance between energy intake and energy expenditure. The prevalence of childhood overweight and obesity has been increasing worldwide over the past few decades, becoming a major public health concern in developed and developing countries, including the Republic of Korea. The prevalence of obesity steadily increased from 8.6\% in 2009 to $10.2 \%$ in 2016 among children aged 2-18 years and from $29.7 \%$ in 2009 to $32.4 \%$ in 2015 among adults in the Republic of Korea. ${ }^{1)}$ Obese children are at greater cardiometabolic risk since they have higher blood pressure, glucose, triglyceride, and low-density lipoprotein cholesterol levels and lower high-density lipoprotein cholesterol levels. ${ }^{2)}$ Childhood obesity often leads to adulthood obesity, which is closely related to cardiometabolic risks such as hypertension, type 2 diabetes, and metabolic syndrome, a major risk factor for increased mortality and morbidity. ${ }^{3)}$

Obesity is a multifactorial chronic disease that results from complex interactions between genetic and environmental conditions. Advances in genetic technology have improved our understanding of the molecular mechanisms of the accumulation of excessive fat by detecting single nucleotide levels using wholeexome, genome, and targeted sequencing techniques. Genetic and epigenetic factors reportedly play a role in the development of obesity. Along with recent technological advances, genetic studies have evaluated the following obesity-related genes: (1) single genes including LEP, LEPR, POMC, MC4R, SIM1, NTRK2, KSR2, CPE, PCSK1, BDNF, SH2B1, and SH2B1; (2) syndromic obesity genes including NIPBL, SH2B1, GNAS, and ALMS1; (3) genes related to overgrowth syndrome, including PTEN, ICR1/
H19/KCNQ1OT1/CDKN1, and RASA1; and (4) miscellaneous genes including MYTL1. ${ }^{4}$ In addition, epigenetic changes, which are reversible chemical modifications to DNA with no changes in DNA sequences, are involved in obesity development through the following mechanisms: (1) DNA methylation (the circadian clock genes [CLOCK, clock circadian regulator; BMAL1, aryl hydrocarbon receptor nuclear translocator-like; PER2, period circadian 2]); (2) histone modification (preadipocyte factor-1 [Pref-1], CCAAT-enhancer-binding protein $\beta$ [C/EBP $\beta$ ], $C / E B P \alpha$, peroxisome proliferator-activated receptor gamma [PPAR- $\gamma]$, and adipocyte protein 2 [aP2]); and (3) microRNAs (miRNA: miR148a, miR-26b, miR-30, and miR-199a). ${ }^{5 \text { ) }}$

Lifestyle-related factors are also related to obesity. The common transition from childhood to adulthood obesity indicates that obese adults are likely to have been obese children. Parents and children usually share a general environment, such as meal patterns and physical activities. In addition to the familial environment, several obesity-related habits of parents that form from childhood will affect their offspring, who will also become obese. Evidence indicates a strong relationship between parental (maternal, paternal, or both) obesity and offspring obesity. ${ }^{6}$ In a very recent issue of Clinical and Experimental Pediatrics, Lee et al. ${ }^{7)}$ reviewed relationship between parental (maternal, paternal, or both) obesity and offspring obesity. Additionally, parental education level, parental smoking status, parental employment status, monthly familial income, and number of household members are closely related to obesity. ${ }^{8)}$

The increasing incidence of childhood and adulthood obesity will threaten public health plans to reinforce the length and quality of life in developed and developing countries. Nations currently face significant challenges to healthcare budgets and will continue to do so. To prevent an increasing prevalence of obesity, it is important to implement weight-reducing programs that include all modifiable factors. It should be remembered that the modifiable factors include siblings and the family as a unit, which can provide the opportunity to comprehensively understand modifiable factors and present a more holistic and effective means to improving childhood obesity. A series of studies indicated that it is more effective to apply weight

Corresponding author: Young Suk Shim, MD. Department of Pediatrics, Ajou University Hospital, Ajou University School of Medicine, 164 World cup-ro, Yeongtong-gu, Suwon 16499, Korea 
reduction programs to families rather than individuals. A few randomized controlled trials revealed that a family-based weight control program (FBP) effectively reduced child and parental obesity. ${ }^{9)} \mathrm{FBP}$, a standard approach to treating obese children, can lead to short- and long-term weight reductions. ${ }^{10)}$ In participating children, FBP improves unfavorable psychosocial outcomes such as depression, lower quality of life, and eating problems, all of which can contribute to worsening obesity. ${ }^{10)}$ To manage childhood obesity, it is important to understand, identify, and modify obesogenic environmental factors using FBP.

In summary, childhood obesity originates from complex genetic and environmental factors. Rapidly growing analytical techniques have contributed to our increased understanding of genetics. A series of studies also provided a comprehensive understanding of obesity-related psychosocial and socioeconomic factors. Obesity in children is closely related to factors in the familial environment, including parental obesity, parental education level, parental smoking, parental employment status, household income, and number of household members. Moreover, family-based strategies are effective. Pediatricians strive to identify all correctable obesity-related family and individual factors and encourage all family members to participate in weight reduction programs.

See the article "Global relationship between parent and child obesity: a systematic review and meta-analysis" via https://doi. org/10.3345/cep.2020.01620.

\section{Footnotes}

Conflicts of interest: No potential conflict of interest relevant to this article was reported.

\section{References}

1. Kim JH, Moon JS. Secular trends in pediatric overweight and obesity in Korea. J Obes Metab Syndr 2020;29:12-7.

2. Lim S, Jang HC, Park KS, Cho SI, Lee MG, Joung H, et al. Changes in metabolic syndrome in American and Korean youth, 1997-2008. Pediatrics 2013;131:e214-22.

3. Llewellyn A, Simmonds M, Owen CG, Woolacott N. Childhood obesity as a predictor of morbidity in adulthood: a systematic review and metaanalysis. Obes Rev 2016;17:56-67.

4. Thaker VV. Genetic and epigenetic causes of obesity. Adolesc Med State Art Rev 2017;28:379-405.

5. Lopomo A, Burgio E, Migliore L. Epigenetics of obesity. Prog Mol Biol Transl Sci 2016;140:151-84.

6. Whitaker RC, Wright JA, Pepe MS, Seidel KD, Dietz WH. Predicting obesity in young adulthood from childhood and parental obesity. N Engl J Med 1997;337:869-73.

7. Lee JS, Jin $\mathrm{MH}$, Lee HJ. Global relationship between parent and child obesity: a systematic review and meta-analysis. Clin Exp Pediatr 2022;65:35-46.

8. Lee HJ, Kim SH, Jin MH, Lee JS. Variability in sociodemographic factors and obesity in Korean children: a cross-sectional analysis of Korea National Health and Nutrition Examination survey data (2007-2015). Ann Epidemiol 2020;43:51-7.

9. Quattrin T, Roemmich JN, Paluch R, Yu J, Epstein LH, Ecker MA. Efficacy of family-based weight control program for preschool children in primary care. Pediatrics 2012;130:660-6.

10. Hayes JF, Fowler LA, Balantekin KN, Saelens BE, Stein RI, Perri MG, et al. Children with severe obesity in family-based obesity treatment compared with other participants: conclusions depend on metrics. Obesity (Silver Spring) 2021;29:393-401.

How to cite this article: Shim YS. Pediatricians must consider familial environment when diagnosing and managing childhood obesity. Clin Exp Pediatr 2022;65:31-2. https://doi.org/10.3345/ cep. 2021.00318 